\section{Socio-naturalezas fluviales en América Latina: Apuntes teórico-metodológicos}

\section{River socio-natures in Latin America: Theoretical-methodological notes}

\author{
Gabriel Garnero \\ Universidad Nacional de Córdoba y CONICET \\ Córdoba, Argentina \\ gabogarnero@gmail.com \\ (iD) 0000-0002-4710-147X
}

\begin{abstract}
Información del artículo
Recibido: 29 abril 2020

Revisado: 6 febrero 2021

Aceptado: 19 febrero 2021
\end{abstract}

ISSN 2340-8472

ISSNe 2340-7743

DOI 10.17561/AT.19.5455

(C) $\mathrm{CC}-\mathrm{BY}$

(c) Universidad de Jaén (España). Seminario Permanente Agua, Territorio y Medio Ambiente (CSIC)

\section{RESUMEN}

Los ríos han tenido una importancia central en la historia de América, desde la llegada de los primeros humanos, hasta el pasado reciente; desde el extremo sur al extremo norte, este y oeste. Corriendo por llanuras y montañas, selvas tropicales y zonas desérticas, llegando al mar o infiltrándose en el corazón del territorio, su presencia ha sido ubicua. Mediante la investigación histórico ambiental de sus influencias, usos, representaciones, conflictos e intervenciones técnicas; daremos cuenta de algunos enfoques desplegados para comprender las mutuas interacciones entre sociedad y agua. En esta presentación comentaré cómo se han construido estas narrativas históricas con eje en dinámicas del agua, su multiescalaridad temporal y espacial, de qué forma la materialidad y los aspectos biofísicos de los ríos han incidido en los desarrollos sociales y la importancia de las relaciones de poder en todas sus manifestaciones.

PALABRAS CLAVE: Ríos, América Latina, Historia ambiental, Agua,

Socio-naturaleza.

\section{ABSTRACT}

Rivers have had a central place in the Americas history, since the arrival of the first humans, until the recent past; from the extreme south to the extreme north, east and west. Running through plains and mountains, tropical jungles and desert areas, reaching the sea or disappearing in the heart of the land, its presence has been ubiquitous. Through historical environmental investigation of its influences, uses, representations, conflicts and technical interventions; we will give an account of some approaches to the understanding of the mutual interactions between society and water. In this presentation, I will review how these historical narratives have been built around water dynamics, their temporal and spatial multiscalarity, how material and biophysical characteristics of rivers have influenced social developments and the importance of power relations.

KEYWORDS: Rivers, Latin America, Environmental history, Water, Socionature. 


\section{Socio-natureza dos rios na América Latina: notas teórico-metodológicas}

\section{RESUMO}

Os rios tiveram uma importância central na história da América, desde a chegada dos primeiros humanos, até o passado recente; do extremo sul ao extremo norte, leste e oeste. Correndo por planícies e montanhas, florestas tropicais e áreas desérticas, chegando ao mar ou se infiltrando no coração do território, sua presença tem sido onipresente. Através da investigação ambiental histórica de suas influências, usos, representações, conflitos e intervenções técnicas; Daremos conta de algumas abordagens implantadas para compreender as interações mútuas entre a sociedade e a água. Nesta apresentação, comentarei como essas narrativas históricas foram construídas com um eixo sobre a dinâmica da água, sua multiescalaridade temporal e espacial, como a materialidade e aspectos biofísicos dos rios influenciaram os desenvolvimentos sociais e a importância das relações de poder. Em todos os seus manifestações.

PALAVRAS-CHAVE: Rios, América Latina, História ambiental, Água,

Socio-natureza.

\section{Les socio-natures des rivières en Amérique} Latine: notes théoriques et méthodologiques

\section{RÉSUMÉ}

Les fleuves ont eu une importance centrale dans l'histoire de l'Amérique, depuis l'arrivée des premiers humains, jusqu'au passé récent; de l'extrême sud à l'extrême nord, est et ouest. Traversant plaines et montagnes, forêts tropicales et zones désertiques, atteignant la mer ou s'infiltrant au cœur du territoire, sa présence est omniprésente. Par l'enquête environnementale historique de ses influences, usages, représentations, conflits et interventions techniques; Nous rendrons compte de quelques approches déployées pour comprendre les interactions mutuelles entre la société et l'eau. Dans cette présentation, je commenterai comment ces récits historiques ont été construits avec un axe sur la dynamique de l'eau, leur multiscalarité temporelle et spatiale, comment la matérialité et les aspects biophysiques des rivières ont influencé les développements sociaux et l'importance des relations de pouvoir. manifestations.

MOTS CLÉS: Rivières, Amérique Latine, Histoire environnementale, Eau, Socio-nature.

\section{La socio-natura dei fiumi in America Latina: note teorico-metodologiche}

\section{SOMMARIO}

I fiumi hanno avuto un'importanza centrale nella storia d'America, dall'arrivo dei primi umani, fino al recente passato; dall'estremo sud all'estremo nord, est e ovest. Attraversando pianure e montagne, foreste pluviali e aree desertiche, raggiungendo il mare o infiltrandosi nel cuore del territorio, la sua presenza è stata onnipresente. Attraverso l'indagine storica ambientale delle sue influenze, usi, rappresentazioni, conflitti e interventi tecnici; Daremo un resoconto di alcuni approcci utilizzati per comprendere le reciproche interazioni tra società e acqua. In questa presentazione, commenterò come queste narrazioni storiche sono state costruite con un asse sulle dinamiche dell'acqua, la loro multiscalarità temporale e spaziale, come la materialità e gli aspetti biofisici dei fiumi hanno influenzato gli sviluppi sociali e l'importanza delle relazioni di potere. manifestazioni.

PAROLE CHIAVE: Fiumi, America Latina, Storia ambientale, Acqua, Socionatura. 


\section{Introducción}

A pesar de numerosos avances y aportes teóricos, las narrativas históricas siguen estando mayormente centradas en actores humanos y gestas sociales, mientras que las dinámicas hidrológicas siguen siendo subestimadas. Para hacer frente a esta dificultad, la historia ambiental y otros enfoques, proponen comprender las relaciones entre sociedades y agua centrándose en las mutuas interacciones. Esto, ha posibilitado avanzar hacia una comprensión más profunda, exhaustiva y equilibrada de los espacios fluviales.

En el marco de estas transformaciones historiográficas, ha quedado en evidencia, que las preguntas e investigaciones abordadas por estudiosos en todo el continente americano, tienen mucho en común y también muestran importantes variaciones. Dentro del marco de la Sociedad Latinoamericana de Historia Ambiental (SOLCHA) se fue conformando un grupo informal de Historia Ambiental de los ríos de América Latina y el Caribe. Nosotros, al igual que muchas otras comunidades de investigación, queremos aportar en la enorme tarea de analizar desde un punto de vista diacrónico, la construcción de nuestros territorios y el rol que han cumplido las corrientes de agua en ese proceso.

Con esa intencionalidad, pretendemos generar estudios ambientales en base a la investigación empírica documental de los usos, representaciones, conflictos e intervenciones técnicas en torno a los cursos de agua en nuestro continente, a lo largo del tiempo. Para ello, hemos evitado que nuestras interpretaciones se limiten a señalar los impactos negativos de los actores humanos sobre una naturaleza prístina, idealizada y dicotómicamente separada de la humanidad o cayendo en interpretaciones ambientales deterministas. En este sentido, hemos avanzado en la exploración de la forma en que las tensiones entre la materialidad fluvial, la técnica y el imaginario de las sociedades constituyeron progresivamente diferentes interacciones entre ríos y seres humanos. Para de esta forma, explicar la construcción de estructuras de poder político, económico y social, así como la configuración de territorios heterogéneos a diversas escalas. Nuestra motivación es presentar trabajos, de diferentes temporalidades y zonas del continente, que den cuenta de algunos de los enfoques sobre historia ambiental de ríos.

Incluimos en nuestra selección, una serie de investigaciones que consideramos suficientemente representativa. Así, el trabajo de Andrea Noria Peña, se sitúa en el Chile colonial. Los trabajos de Carolina Ardila Luna,
Javier Bonilla, y Rebeca López Mora, sitúan sus historias en Colombia, Panamá, y México respectivamente y abarcan una larga temporalidad, desde el período XV hasta el siglo XX. El trabajo de Gabriel Garnero focalizado en el período de consolidación del Estado Nacional Argentino, hasta principios del siglo XX. Y finalmente, el de Luisa Prazeres-Vasconcelos y Felipe Eduardo Ferreira-Marta, de Brasil, que analiza procesos de la segunda mitad del siglo XX. Como puede apreciarse, las narrativas van desde la colonia -portuguesa y española- hasta el pasado reciente, desde el extremo sur al extremo norte, este y oeste de Latinoamérica. Asimismo, en las historias incluidas, recorremos llanuras y montañas, selvas tropicales y zonas desérticas, ríos que llegan al mar y ríos enclavados en el corazón del continente. De esta forma, creemos que ofrecemos gran diversidad y representatividad, como para constituir un aporte interesante a la historiografía ambiental latinoamericana. A pesar de ello, la selección constituye solo una muestra de la multiplicidad de investigaciones sobre el tema que se están realizando a nivel continental, desde gran variedad de perspectivas.

Consideramos que reunir investigaciones disímiles, basadas en las premisas de la historia ambiental y con objeto en los ríos, constituye un ejercicio modesto, aunque sumamente necesario. Como comentamos previamente, la temática que nos convoca es una de gran amplitud y han existido numerosas formas de abordarla. Por ello, el objetivo de esta presentación, es delinear algunos aspectos teórico-metodológicos respecto a las historias de ríos y del agua, que nos permitan vincular los diversos trabajos incorporados al dossier. En las sucesivas páginas, comentaré cómo se han construido narrativas históricas con eje en dinámicas del agua, la multiescalaridad temporal y espacial de estas historias, de qué forma la materialidad y los aspectos biofísicos de los ríos han incidido en los desarrollos sociales y la importancia de las relaciones de poder en todas sus manifestaciones. Finalmente, concluiré la presentación, resaltando el rol de la tecnología, y cómo su conjunción con los otros factores analizados, movilizan procesos de coproducción de socionaturalezas fluviales, que la Historia Ambiental de los ríos se encarga de estudiar. Creo que estos elementos, en términos generales, serán balizas que nos posibilitarán recorrer y comparar los diferentes casos empíricos presentados. 


\section{Las narrativas fluviales en la historia ambiental: Pasado, presente y futuro}

El primer aspecto común de las investigaciones aquí presentadas, es su carácter historiográfico, cuyo resultado adopta forma narrativa. Estas construcciones, empíricamente informadas, constituyen el principal aporte de la disciplina histórica al debate ambiental contemporáneo. Así, permiten dar sentido y estructurar múltiples causalidades interaccionando -en el espacio y a lo largo del tiempo- para reconstruir las diversas capas de cambio en el vínculo entre sociedad y la biofísica del agua ${ }^{1}$.

La necesidad de respaldo empírico, implica incorporar a los análisis ambientales, información proveniente de numerosas fuentes documentales primarias. En este sentido, es menester evitar descripciones ecológicas ahistóricas o anacrónicas, usando arbitrariamente y superficialmente la documentación ${ }^{2}$. El análisis de fuentes heterogéneas, nos permitió reconstruir aquellos espacios fluviales que constituyen el objeto de nuestras narrativas ${ }^{3}$. Entre ellas, se cuentan fuentes primarias y secundarias coloniales, desde el siglo XV al XIX, tales como disposiciones institucionales -Actas de cabildos, Real Audiencia, Archivo General de Indias- entre otros. Para períodos posteriores, se utilizaron documentos de los gobiernos nacionales que los sucedieron, tales como actas de sesiones de órganos legislativos, disposiciones administrativas e informes de reparticiones técnicas, tanto a nivel local, como regional y nacional. Sumado a esto, se analizaron numerosas publicaciones periódicas y especializadas. Se destacó también el uso de escritos de viajeros, cronistas e incluso fuentes historiográficas orales, para períodos más recientes. En todos los casos, se evidencia un amplio acceso e investigación, en diversos repositorios, archivos y bibliotecas, en múltiples escalas geográficas.

Las historias de ríos, han tenido un rol protagónico en el desarrollo de la historiografía ambiental. ${ }^{4}$ Este rol no fue estático, si no que se produjeron cambios en la forma de conceptualizarlos y abordarlos. Inicialmente, predominaron narrativas declesionistas, sobre degradación, explotación y conquista del agua ${ }^{5}$. A pesar de los indudables méritos de esas narrativas, actualmente emergen otras metodológicamente más sofisticadas,

\footnotetext{
1. Wesselink, Kooy, y Warner, 2017, 10.

2. Sánchez, 2018.

3. Mauch y Zeller, 2008, 6. Winiwarter, Schmid, y Dressel, 2013, 111.

4. Worster, 1992. Febvre, 2004. Winiwarter, Schmid, y Dressel, 2013.

5. Jakobsson, 2002. Winiwarter, Schmid, y Dressel, 2013. Schönach 2017.
}

que incorporan nuevas facetas de las relaciones sociedad-río y que se articulan con desarrollos en otras disciplinas ${ }^{6}$. Un pivote en esta transformación historiográfica, es la reconsideración sobre selección de puntos iniciales y finales. La definición de estos marcadores, no es casual y delimita fuertemente la moral de las historias ${ }^{7}$. Permite a los lectores identificar actores y circunstancias; procesos de toma de decisiones; causalidades; continuidades y rupturas; éxitos o fracasos. La construcción historiográfica, está sumamente influenciada por estas decisiones.

Además de su impacto historiográfico, las construcciones histórico ambientales en torno a los ríos, tienen un papel importante que cumplir en la comprensión de las interacciones socionaturales fluviales en general ${ }^{8}$. A pesar de la imposibilidad de predecir, ofrecen parábolas sobre cómo interpretar lo que pueda suceder, teniendo esto profundas implicancias políticas y sociales ${ }^{9}$. Pueden colaborar en la distinción de responsabilidades, en la dirección de políticas o en la prevención de decisiones inapropiadas, antidemocráticas o ambientalmente insostenibles ${ }^{10}$. Además, las historias en torno al agua, expresan aspectos no dichos de otros procesos históricos más generales y dan voz a actores anteriormente relegados. En este sentido, no solo representando a la naturaleza, que no habla; sino también a sectores menos favorecidos, colonizados, dominados, vencidos. Todos ellos, tradicionalmente acallados en nombre del progreso de nuestro continente ${ }^{11}$. Así, las narrativas en torno a los ríos Grande, Meta, Los Sauces, Mapocho, Tlalnepantla y Verruga- que aquí exhibimos- constituyen una forma de dotar de voz no solo a estas entidades, sino también a todos los grupos y sectores sociales que se vincularon de alguna forma con ellos en su transcurrir temporal.

\section{Múltiples escalas de los ríos: espaciales y temporales}

Un segundo elemento en común en nuestras historias de ríos, es el replanteo en torno a las escalas. Las definiciones temporales y espaciales han sido siempre un aspecto importante en la Historia, puesto que permiten dar cuenta de los procesos estudiados. Generalmente, los historiadores recurrieron a divisiones administrati-

\footnotetext{
6. Swyngedouw, 1999. Winiwarter, Schmid, y Dressel, 2013, 104. Robbins, Hintz, y Moore, 2014.

7. Cronon ,1992, 1349. Mauch y Zeller, 2008, 60.

8. Martos-Núñez y Martos-García, 2015, 122.

9. Cronon, 1993, 43.

10. Mauch y Zeller, 2008, 62. Robbins, 2011, 132.

11. Leff, 2005, 27.
} 
vas, políticas o pretendidas similitudes socioculturales. En el caso del tiempo, se han ceñido a divisiones basadas en procesos sociales, políticos o económicos. Todas ellas son escalas relativamente estables, fundamentalmente fijas y concebidas como predeterminadas ${ }^{12}$. Aunque siempre fue necesario analizarlas críticamente, el intento de incorporar las dinámicas naturales en los estudios históricos, lo tornó imperativo ${ }^{13}$.

Un gran avance, es su resignificación en términos relacionales. Las escalas no son naturales, sino que son producidas, a través de las interacciones diacrónicas entre prácticas sociales, procesos ambientales y fuerzas estructurales ${ }^{14}$. A la vez, no son totalmente fijas, ni totalmente fluidas; varían en la medida en que son construidas y reconstruidas a través de ideas, creencias y suposiciones; que son la base de prácticas concretas discursivas, ideológicas, culturales, científicas y materiales $^{15}$. Esta compleja articulación escalar, emerge de las dinámicas de materiales, energía y capital, inherentes a lo que muchos denominan procesos de metabolismo socioecológico y los niveles de regulación y gobernanza en el que están incorporados ${ }^{16}$.

\section{Dimensiones espaciales}

A la Historia le ha resultado difícil explorar la dimensión espacial de los fenómenos que estudia, saliendo de su autoproyección como ciencia del tiempo ${ }^{17}$. En las narrativas fluviales, como las que aquí compilamos, esto se torna imprescindible. Las divisiones jurisdiccionales tradicionales, raramente coinciden con la forma en que las dinámicas del agua y los ríos pueden ser mejor explicadas $^{18}$. En este sentido, no existe una correspondencia exacta entre los límites hidrológicos y políticos, incluso entre fenómenos diversos ocurriendo dentro de cada una de las grandes esferas de causalidad. Asimismo, las historias en torno a cambios socioambientales fluviales, pueden y deben ser escritas considerando diferentes niveles, entre lo micro y lo macro, entre lo local y lo global y las interacciones existentes entre ellos ${ }^{19}$. La elección de la escala espacial, es crucial para evaluar las transformaciones ambientales y los fenómenos e inte-

\footnotetext{
12. Brenner, 1998. Gallini, 2004, 3-4.

13. Arnold y Elier, 2000, 60. Gallini, 2004, 3. Swyngedouw, 2010, 5.

14. Smith, 1994. Norman, Bakker, y Cook, 2012. Boelens et al., 2016, 5.

15. Menga y Swyngedouw, 2018, 4.

16. Swyngedouw, 2010, 9.

17. Gallini, 2004, 3.

18. Mosley, 2010, 3

19. Mosley, 2010, 3. Walker, 2011. Schönach, 2017, 12.
}

racciones responden a procesos espacialmente situa$\operatorname{dos}^{20}$.

En las historias de ríos, ha ganado peso el concepto de cuenca hidrográfica como unidad de análisis. Esta idea es útil, porque puede incluir aspectos biofísicos e incorporar acciones humanas que se asientan sobre el territorio ${ }^{21}$. Sin embargo, no es necesariamente siempre correcto y aplicable a todos los casos. Se le ha criticado su insuficiente desarrollo teórico y su emergencia se asocia a intenciones de control tecnocrático ${ }^{22}$. En este sentido, la noción de cuenca también es un constructo político e ideológico, con sus representaciones y justificaciones ${ }^{23}$. Como se ve, aún aquellas escalas que aparecen como soluciones, no pueden tomarse acríticamente.

En las trayectorias de los ríos que aquí aparecen, puede apreciarse el cuidado en considerar diversas escalas espaciales. En este sentido, todos los trabajos destacan la influencia que los ríos tuvieron en la organización territorial. Durante el período colonial, como expresa Noria Peña, fueron conectores y estructuradores de centros urbanos y ruralidades, espacios predilectos para las fundaciones españolas y portuguesas. En este mismo sentido, principalmente, los trabajos de Ardila Luna y Bonilla, resaltan las posibilidades de navegación y transporte en redes interregionales e internacionales. El río Meta, uniendo montaña con llano y el Grande en el proyecto de unión del océano Pacifico con el Atlántico. Esto resalta la dimensión espacial vinculante de los espacios fluviales -a nivel local, regional e internacional- y su contribución en explicar la configuración de redes de conexión entre diferentes zonas. En los análisis del dossier, se han considerado las dimensiones espaciales de las historias y sus múltiples escalas, tanto administrativas y sociopolíticas, como la construcción conceptual de cuencas y la articulación entre ellas.

\section{Dimensiones temporales}

Así como discutimos las escalas espaciales, también hay que considerar las múltiples temporalidades fluviales. A diferencia de los procesos espaciales, el eje de la disciplina ha estado tradicionalmente enfocado en esta dimensión de la existencia. No obstante, sigue siendo

\footnotetext{
20. Bakker, 2012, 525. Boelens et al., 2016, 6 .

21. Cabral, 2007. Arruda, 2015.

22. Molle y Wester, 2009. Cohen y Davidson, 2011.

23. Molle, 2009, 484.
} 
dudoso determinar qué hechos y de qué duración provocan realmente cambios significativos y duraderos ${ }^{24}$.

Múltiples esferas de incidencia, subsumen a su vez diversidad de temporalidades, dando lugar a un espectro muy diversificado ${ }^{25}$. Por un lado, dinámicas ecológicas y ciclos naturales asociados a los ríos, tienen diferentes ritmos, intensidad y duración. No son iguales los ciclos de la fauna acuática, que los ritmos climáticos o geomorfológicos, que pueden oscilar de días a siglos, por dar algunos ejemplos. Por otro lado, lo mismo ocurre con los procesos sociales, como problematizó ya Braudel con sus múltiples tiempos que conforman el pasado $^{26}$. De este modo, diferentes historias fluviales, pueden incorporar capas de análisis temporales muy diversas. El proceso de definición del objeto estará profundamente determinado por la dinámica e interjuego de estas escalas.

Estas reflexiones, aparecen insistentemente en las investigaciones desarrolladas. El trabajo de Noria Peña, se centra exclusivamente en el período colonial, insuficientemente trabajado tradicionalmente ${ }^{27}$. Sin embargo, tienen gran protagonismo las estaciones, precipitaciones y otros aspectos biofísicos asociados al agua, con sus ciclos específicos. Garnero, también hace jugar múltiples tiempos, pero destacando el período del siglo XIX en el qué, usando palabras de Bonilla, se produce una "ruptura metabólica" en las relaciones sociedad-naturaleza. Ardila Luna, Bonilla y López Mora, utilizan un enfoque de larga duración, para poder apreciar otros tipos de continuidades y rupturas. Por otra parte, Prazeres-Vasconcelos y Ferreira-Marta, entrelazan en su estudio las temporalidades de la vida de un hombre, con los ritmos de transformación de una ciudad y las dinámicas del río. Como vemos, todos ellos, además de abarcar períodos diferentes, hacen interaccionar distintas escalas temporales de los procesos analizados. En definitiva, la articulación multiescalar espacial y temporal, nos sirve para prevenirnos de aquellas interpretaciones ambientales fuertemente ahistóricas o que desestiman la complejidad espacial ${ }^{28}$.

\footnotetext{
24. Arnold y Elier, 2000, 60.

25. Hughes, 2009, 8.

26. Cronon, 1993, 10.

27. Soluri, Leal, y Pádua, 2018, 13:4.

28. Cronon, 1993, 10.
}

\section{Esferas de causalidad en las historias fluviales: el rol de la materialidad}

En tercer lugar, como se ha delineado sucintamente en el anterior apartado, un aspecto clave que las historias fluviales aquí reunidas reflejan, tiene que ver con el rol de la materialidad. La conjugación de diversas esferas de causalidad, vinculadas tanto a aspectos sociales como naturales de la existencia, ha generado largos debates en el seno de las disciplinas interesadas en superar la dicotomía entre materialismo/culturalismo ${ }^{29}$. La Historia, también ha mostrado un renovado interés en reintroducir enfoques materialistas para analizar las interacciones humanas en el pasado ${ }^{30}$.

Las historias de ríos, han tenido que incorporar la materialidad y definir su grado de incidencia en otros procesos. La expresión física del mundo es compleja, dinámica y por lo tanto, difícil de conocer ${ }^{31}$. En nuestro caso, no se refiere solo al agua, sino que implica considerar el rol de otras características biofísicas y ecológicas. Y adicionalmente, el resto de "objetos" (tecnología) que hacen diferencia en el desarrollo de percepciones, construcciones discursivas y respuestas humanas; y que no son solo simples substratos predeterminados ${ }^{32}$.

\section{Descentrar la agencia humana}

La reconsideración de la materialidad, repercute en la forma de conceptualizar los roles protagónicos en nuestras narrativas. Produce un descentramiento o redistribución de la agencia, anteriormente enfocada en las acciones humanas ${ }^{33}$. Esto ha llevado a preguntarse si la agencia de las cualidades materiales de un río, tienen status similar a la de los actores sociales o hasta qué punto ayudan a explicar resultados sociales; o si al estudiarlos de este modo, seguimos haciendo Historia ${ }^{34}$. Los interrogantes sobre la materialidad, vienen asociados a cuestionamientos sobre su "naturalidad". Algunos, postulan que las intervenciones humanas sobre los ríos son capaces de desproveerlos de ese carácter $^{35}$. Otros, cuestionan directamente el pretendido carácter "natural" del agua ${ }^{36}$.

\footnotetext{
29. Sörlin y Warde, 2005, 16.

30. Cronon, 1993, 38. Swyngedouw, 2010, 11. Blackbourn 2011, 19. Gallini, 2012, 390.

31. Simmons, 2008, 2.

32. Bakker y Bridge, 2006, 19. Bakker, 2012.

33. Bakker, 2012, 621.

34. Robbins, 2011, 131.Blackbourn, 2011, 20.

35. Tvedt, 2010, 152

36. Wesselink, Kooy, y Warner, 2017, 7.
} 
A pesar de diferencias y disquisiciones, es indudable que la faceta física de la existencia, es un factor que incide en las transformaciones sociales. La materia, asimismo, presenta resistencia a prácticas humanas que se le ejercen; así, producir o mantener configuraciones materiales requiere capacidad de trabajo y su sostenimiento a lo largo del tiempo ${ }^{37}$. Estos procesos dialécticos, de coevolución histórica de configuraciones materiales y prácticas, tienen que ser también objeto de atención de los historiadores.

Por razones obvias, en las narrativas fluviales, uno de los factores protagonistas en la esfera material de causalidad, es el agua y sus dinámicas. Ella actúa y es a su vez es afectada por humanos y otras especies, infraestructura, ciclos biogeoquímicos, entre otros ${ }^{38}$. Así, el agua cambia de ser objeto de procesos sociales, a ser transformada y transformadora de relaciones sociales, estructuras y subjetividades ${ }^{39}$. El estudio de ríos, involucra analizar los flujos de agua y cómo estos, en su interacción con otras esferas de causalidad -en el tiempo y el espacio- han producido y reproducido estructuras que impactan en las acciones humanas, territorios y sistemas sociales ${ }^{40}$.

Al incorporar todas estas esferas de causalidad hay que precaverse de no caer en alguno de los diferentes tipos de determinismos ${ }^{41}$. Esto incluye el tradicional determinismo geográfico, pero también el reduccionismo ambiental, de algunos ecólogos humanos o sociobiólogos, que pretenden explicar toda dinámica social por medio de leyes ecológicas ${ }^{42}$. Por el contrario, sistemas culturales y ambientales interactúan sin que ninguno determine por completo el resultado ${ }^{43}$.

En nuestros trabajos, la "resistencia de la materialidad" se plasma de muchas formas.

Especialmente Noria Peña, pero también Bonilla, Garnero, López Mora, Prazeres-Vanconcelos y FerreiraMarta muestra la forma en que la materialidad de los espacios fluviales se percibió como amenaza. Así, procesos naturales extremos, como lluvias torrenciales e inundaciones, han hecho que el agua y los ríos fueran a veces conceptualizados como enemigos o fuentes de peligro. Sin embargo, períodos de menos precipitaciones, también han sido muestra de aquella resistencia. Características biofísicas - como caudales, geomorfología

\footnotetext{
37. Smith, 1994. Fischer-Kowalski y Erb, 2016, 46.

38. Schmidt, 2014, 221.

39. Linton y Budds, 2014, 170.

40. Tvedt, 2010, 156. Linton y Budds, 2014, 170.

41. Menga y Swyngedouw, 2018, 18.

42. de Molina y Toledo, 2014, 3:20.

43. Cronon, 1993, 38. Menga y Swyngedouw, 2018, 11.
}

o clima- posibilitaron u obstaculizaron su uso como vías de comunicación; como puede verse especialmente en el caso de Ardila Luna y Bonilla. O su ubicación geográfica y régimen de precipitaciones permitió o limitó la ejecución de sistemas de riego, aprovisionamiento urbano o provisión de energía hidroeléctrica. En este sentido, las cuencas del río Meta, Grande, de Los Sauces, Mapocho, Tlalnepantla y Verruga interactuaron con otras esferas de causalidad y son coautoras de nuestras historias. Adicionalmente, no podemos olvidar que las intervenciones humanas materializadas en estos espacios fluviales, también se constituyeron en elementos activos, posibilitando o resistiendo futuras transformaciones.

\section{Poder, Estado y conflictos en las historias de ríos}

En cuarto lugar, las pesquisas compiladas, destacan el rol central del poder en las interacciones socionaturales. Esta consideración, tiene larga trayectoria en el análisis histórico de los sistemas fluviales. Estudios como el de Wittfogel sobre el despotismo hidráulico, dieron lugar a profundos debates sobre la forma en que el control de las dinámicas fluviales, ha sido también expresión de poder político ${ }^{44}$. Las entidades estatales, no deben entenderse como configuraciones fijas y predeterminadas, sino como procesos situados (temporal y espacialmente) y el estudio de su dinámica de co-construcción con los sistemas fluviales, contribuye a interpretarlos ${ }^{45}$. Así, por un lado, tenemos el mundo biofísico, o esfera de causalidad material, generadora de multiplicidad de "flujos" ${ }^{46}$. Y por el otro lado, entidades políticas - conjunto de instituciones, leyes y prácticasque hacen esfuerzos constantes para domesticarlos ${ }^{47}$.

Con esta idea en mente, numerosas narrativas fluviales se centraron en la construcción y operación de grandes sistemas hidráulicos por parte de administraciones políticas centralizadas, tanto en antigüedad como en el pasado reciente ${ }^{48}$. Pues si bien la infraestructura hidráulica no determina la existencia de una burocracia centralizada, el poder ejercido sobre las dinámicas hídricas mediante la expansión de estos

\footnotetext{
44. Wittfogel, 1957.Worster, 1992. Banister, 2014. Worster, 1992, 20. Frioux, 2014, 138. Gómez-Espín, 2019.

45. Menga y Swyngedouw, 2018, 6 .

46. Banister, 2014, 208. Fischer-Kowalski y Erb, 2016.

47. Scott, 1998.

48. Wittfogel, 1957. Hunt, 2009, 6. Worster, 1992. Solomon, 2010, 39. Molle, Mollinga, y Wester, 2009.
} 
sistemas -riego, saneamiento, energía hidráulica, entre otros- ha provisto medios para que, dentro de grupos humanos, algunos sectores asentaran su poder sobre otros ${ }^{49}$.

De este modo, el acelerado proceso de emergencia y consolidación de Estados Nación modernos a partir del siglo XIX implicó grandes transformaciones en los espacios fluviales. La cooperación entre autoridades y planificadores en estos regímenes, para producir y regular esquemas de ingeniería social y natural a gran escala, constituye una dimensión medular de nuestras historias de ríos ${ }^{50}$.

Por un lado, los trabajos evidencian la relación entre dinámicas del agua y el régimen colonial ibérico en sus múltiples localizaciones y facetas, punto comparativo cardinal en la historia ambiental latinoamericana ${ }^{51}$. En el caso de Noria Peña, se muestra la relevancia de las intervenciones de las autoridades coloniales en Chile y sus prerrogativas en la atención de la amenaza que implicaba el Mapocho. Asimismo, el pasado colonial común, constituye una plataforma para analizar la forma en que las entidades políticas latinoamericanas independientes, se vincularon con sus espacios fluviales, durante sus distintivos procesos de consolidación territorial. Estas intervenciones, estuvieron asociadas a legitimar las nuevas configuraciones de poder, los nuevos regímenes y fueron símbolo del estatus nacional ${ }^{52}$. Así, emergieron proyectos que replicaban en Latinoamérica las ideas higienistas de sanidad y modernización urbana emergidas en Europa, como muestra el caso del río Verruga, en Brasil. Otras, de acondicionamiento para transformar los ríos en vías fluviales ligadas a los mercados internacionales, como el Meta, en la republica Colombiana, o grandes planes adecuación de espacios fluviales en vista de sostener el ideal transitista, como en el caso panameño. También avanzaron proyectos multifacéticos de control federal sobre el agua de consumo e irrigación, como los casos del río de Los Sauces y Tlalnepantla en Argentina y México. Estos procesos han sido definidos por algunos estudiosos, como una "misión hidráulica” del Estado, caracterizada por el crecimiento de burocracias especializadas o hidrocracias ${ }^{53}$. En definitiva, las narrativas históricas, hallan espacio fructífero para el análisis y la comparación en el proceso paralelo de conformación multiescalar espacial y

\footnotetext{
49. Worster, 1992, 50. Radkau, 2008, 91.

50. Scott, 1998. J. R. McNeill, 2005, 157. McCully, 2004, 1.

51. Soluri, Leal, y Pádua, 2018, 13:5.

52. McNeill, 2000, 157. Simmons, 2008, 10.

53. Wester, 2009, 10. Molle, Mollinga, y Wester, 2009, 336.
}

temporal, de centralización política y de control sobre las dinámicas fluviales ${ }^{54}$.

\section{Agua y las dimensiones del poder}

No obstante, el entrelazamiento entre dinámicas de poder y agua, trascienden el estudio de la centralización política. Esta fuerza no puede considerarse una entidad monolítica y estatal que se ejerce solo de arriba hacia abajo; si no que todas las personas, inevitablemente, actúan en campos de poder ${ }^{55}$. Su presencia se visualiza de forma descentralizada, penetrante y productora; que contribuye a explicar las interacciones y transformaciones en los sistemas fluviales ${ }^{56}$. El entrelazamiento entre agua, poder y dinámicas sociopolíticas de forma más transversal, permite construir e interpretar las diferentes escalas espaciales y temporales ${ }^{57}$. Asimismo, en cada sociedad, la movilización de agua para diferentes usos, en diferentes momentos y lugares, da cuenta de configuraciones y distribuciones de poder heterogéneas ${ }^{58}$. Estos entrelazamientos y constituciones de poder distintivas en torno al agua, también son el eje de nuestras historias fluviales.

Los conflictos en torno a los ríos, son un tipo de exteriorización de aquellas dinámicas de poder y, por lo tanto, sumamente relevantes en nuestras narrativas ${ }^{59}$. Las disputas en torno a la escasez, por ejemplo, son socialmente producidas; cuando algunos sectores - autoridades estatales, empresas privadas o élites- erigen barreras políticas, tecnológicas o económicas, para apropiarse o limitar el acceso al agua. ${ }^{60}$ Asimismo, estas restricciones, pueden acelerar rivalidades entre grupos sociales subalternos (en base a género, clase o étnia) ${ }^{61}$. También debemos tener en consideración la miríada de dinámicas biofísicas -climáticas, químicas, biológicas, geolmorfológicas, entre otras- asociadas a los sistemas fluviales, que dependiendo el caso, pueden incidir en el desencadenamiento de antagonismos sociales ${ }^{62}$. En sus diversas manifestaciones y formas, los conflictos crean nuevos paisajes, son un factor de dinamismo y transformación en las relaciones socioecológicas. El enfoque del

\footnotetext{
54. Wester, 2009, 27.

55. Robbins, Hintz, y Moore, 2014, 124.

56. Swyngedouw, 2009, 57. Robbins, Hintz, y Moore, 2014, 124. Rattu y Véron, 2016, 153. Obertreis et al. 2016, 173.

57. Swyngedouw, 2010, 12. Obertreis et al. 2016, 173. Menga y Swyngedouw, 2018, 12.

58. Swyngedouw, 2009, 57

59. Schönach, 2017, 12. Latta y Sasso, 2014. Ferreyra, 2017.

60. Molle y Wester, 2009, 3

61. Le Billon ,2015, 599-600. Frioux, 2014, 124.

62. Le Billon, 2015, 599.
} 
conflicto, ha sido una forma de superar la dicotomía sociedad naturaleza; dado que puede revelar la estructura de poder materializada ${ }^{63}$.

La idea de la presencia del poder como fuerza trasnversal y su exteriorización conflictiva, es clara en los trabajos reunidos. En este sentido, las historias representan multiplicidad de actores y grupos accionando en múltiples direcciones. En López Mora, Noria Peña y Garnero, por ejemplo, los desacuerdos sobre cómo protegerse de la amenaza del río o cómo repartir el agua, desencadenaron acciones por parte de autoridades locales, regionales e incluso nacionales. Estas fuerzas de abajo hacia arriba, ejercidas por grupos indígenas, afrodescendientes, criollos, campesinos, entre otros, impulsaron regulaciones, construcción o modificación de obras y muchas veces resistieron las iniciativas desde los sectores poderosos.

\section{Espacios fluviales, tecnología y modernización}

En quinto lugar, es esencial en nuestras historias, la consideración en torno a la ciencia, la tecnología y la idea de progreso. La emergencia de los Estados Nación modernos y el enorme incremento de su capacidad de intervención sobre las dinámicas hidrológicas, está ligada a sus pretensiones modernizantes y al incremento de su capacidad tecnológica ${ }^{64}$. El compromiso con la razón instrumental, la emergencia de la ciencia moderna y sus derivaciones prácticas, subordinaron a la naturaleza y amplios sectores sociales a procesos de cambios continuos; caracterizados por relaciones de poder y mecanismos estructurados en torno a ideales de progreso y desarrollo. ${ }^{65} \mathrm{El}$ nuevo régimen industrial, se asoció a un crecimiento físico sin precedentes, tanto en términos de población como en consumo material per cápita; que se expresó en procesos de marcada urbanización, industrialización, intensificación agrícola e incremento de la actividad económica en general ${ }^{66}$.

Los espacios fluviales, fueron un elemento central en todos estos procesos, que llegaron a alterar el agua en su dimensión conceptual ${ }^{67}$. La coexistencia de múltiples ideas a la que había estado asociada, dio paso a

\footnotetext{
63. Armiero, 2008, 61.

64. Scarpino, 2014, 165.

65. Worster, 1992, 56. Swyngedouw, 1999.

66. Stewart, 1998, 357. Krausmann, Weisz, y Eisenmenger, 2016, 86. López, 2016. Gierlinger et al., 2016. Schönach, 2017, 13.

67. Bakker, 2012, 618.
}

un paradigma monolítico, que la convirtió en H2O; sustancia química unívoca, commodity para ser controlada y utilizada ${ }^{68}$. Simultáneamente, la transición desde un paradigma miasmático de la salud a uno microbiano, coadyuvó en disparar la demanda de su suministro constante en las crecientes ciudades ${ }^{69}$.

Aquellas transformaciones, se asociaron con tecnologías hidráulicas e infraestructura específica. Es decir, nuevas configuraciones entre artefactos técnicos, marcos regulatorios, normas culturales, flujos ambientales, mecanismos de financiamiento, formas de gobernanza, entre otros ${ }^{70}$. Estos elementos tienen un rol productivo en el surgimiento de espacios y entidades entremezcladas, oficiando de punto de unión entre interacciones sociales y la naturaleza ${ }^{71} \mathrm{La}$ forma particular en que se ensamblan en lugares y momentos específicos, forman un engranaje más de los procesos de transformación fluvial ${ }^{72}$.

En casi todas las investigaciones presentadas, el ascenso del pensamiento antropocéntrico, nuevos paradigmas científicos y tecnologías hidráulicas en los países occidentales, ocupa un lugar predilecto. Cómo expresa Bonilla, los desarrollos científicos y tecnológicos y la reducción de la naturaleza no humana a reserva de posibles mercancías, fueron extrapolados e imitados por los sectores dirigentes y cuadros técnicos en las nuevas naciones latinoamericanas a partir del siglo XIX. Este impacto, se evidenció en la multiplicación de grandes proyectos de transformación a gran escala de los sistemas fluviales; con el objetivo de producir una modernización acelerada. Los trabajos de Bonilla, Garnero, López Mora, Prazeres-Vasconcelos y FerreiraMarta, muestran como arquitectos, ingenieros y técnicos, nacionales o extranjeros, fueron portadores de saberes y autoridad para materializar las grandes proyecciones. De esta forma el agua, fue mero insumo en la "nueva escalera acuática" de Panamá; una "fonte de abastecimento" para el caso de Brasil, una carretera de colonización para el de Colombia; insumo agrícola y energía potencial, para los casos mexicano y argentino. Pero, lo que en todos los casos tiene en común, es que, ligada a nuevas tecnologías, constituyó un "instrumento civilizatorio". Es decir, una herramienta esgrimida para forzar la entrada de sociedades latinoamericanas

\footnotetext{
68. Worster, 1992, 52. Hamlin, 2000, 315.; Molle y Wester, 2009, 5; Hassan,2010, 46.

69. Rautenen et al., 2010, 190. Mora, 2019.

70. Hassan, 2010, 42. Kaika, 2006, 276.

71. Castro, 1995, 31. Mosley, 2010, 2. Agnoletti y Serneri, 2014, 4:VII. Schönach, 2017, 14. Mosley 2010, 2. Agnoletti y Serneri 2014, 4:VII.

72. Barco, 2013. Rückert 2017, 158. Obertreis et al., 2016, 172.
} 
consideradas periféricas, atrasadas o "bárbaras"; dentro de un régimen moderno de orden y progreso.

\section{Coevolución: Sistemas hidrosociales, socio-naturalezas, sistemas hídricos, waterscapes}

Finalmente, es hora de armar el rompecabezas. La consideración de las diferentes esferas de causalidad y procesos que hemos abordado y la compleja dinámica entre ellas - a lo largo del tiempo y del espacioconstituyen el eje de las historias fluviales. La mayoría, discute ahora en términos dialécticos los intercambios entre dinámicas biofísicas e intervenciones humanas y las transformaciones que experimentan temporal y espacialmente ${ }^{73}$. Estos elementos vinculados -humanos, tecnología y ambiente- forman parte de un continuum, en lugar de ser compartimentos estancos ${ }^{74}$. Su relación puede considerarse interna, dentro de sistemas o configuraciones híbridas entre cultura, comunicación y mundo material ${ }^{75}$. Asimismo, las determinaciones entre tecnología, ecología y sociedad son recíprocas, de forma no hegemónica y evitando así, formas deterministas en algún sentido ${ }^{76}$.

Existen distintos abordajes sobre la complejidad de estas interacciones. Los miembros de la escuela de ecología social de Viena, por ejemplo, hablan de sitios socionaturales, como nexos entre las prácticas humanas por un lado y su organización o configuración material en la otra ${ }^{77}$. Y relacionado con esas ideas, González de Molina y Toledo, dan especial importancia al análisis de la dimensión metabólica de toda acción humana, es decir, las implicancias que estas tienen en términos energéticos y materiales ${ }^{78}$. Otros investigadores, como Tvedt, destacan el concepto de sistema hídrico y la necesidad de deconstruir analíticamente grandes términos como naturaleza, ambiente o ecosistema, en variables más manejables ${ }^{79}$. Esto, hace posible estudiar la relación entre sociedad y naturaleza y entre aquellos elementos dentro de ambas esferas, tanto diacrónica como sincrónicamente. Finalmente, también destaco los estudios de los espacios fluviales como territorios hidrosociales.

\footnotetext{
73. Swyngedouw 1999. Mauch y Zeller, 2008, 7.

74. Mauch y Zeller, 2008, 7 .

75. Fischer-Kowalski y Haberl, 2007, 8. Linton y Budds, 2014, 176. Wesselink, Kooy, y Warner, 2017, 7.

76. Bakker, 2012, 619. Boelens et al., 2016, 3. Winiwarter et al., 2016, 150.

77. Fischer-Kowalski y Weisz, 2016. Winiwarter y Schmid, 2020, 35.

78. de Molina y Toledo, 2014, 3:23.

79. Tvedt, 2010, 148
}

Cuyos límites naturales y sociales son conceptualizados y materializados por medio del interjuego entre prácticas humanas; flujos de agua; tecnologías hidráulicas; elementos biofísicos; estructuras socioeconómicas e instituciones culturales y políticas ${ }^{80}$.

Todos estos enfoques, tienen en común la idea de que los flujos de agua, participan activamente en procesos creativos - co-construcción, co-producción, entre otros- de socionaturalezas (ver Figura 1$)^{81}$. Los espacios así constituidos, ya se vean como sistemas hídricos, sitios socio-naturales, "waterscapes" o territorios hidrosociales, no son fijos, inmutables y predeterminados; sino que están íntimamente ligados a las sociedades que los formaron y materializan sus contradicciones, conflictos y luchas ${ }^{82}$.

Nuestros diversos trabajos, articulan sus narrativas en torno nociones coevolutivas, como las reseñadas anteriormente. En este sentido, Noria Peña analiza la construcción social de un espacio fluvial signado por el riesgo, y cómo se convirtió en un geosímbolo de la sociedad Santiaguina colonial. Ardila Luna, por su parte, analiza el río Meta, y la forma en que el entrelazamiento de discursos y prácticas lo configuró en un territorio de "frontera". Asimismo, en Bonilla, el corredor interoceánico de Panamá, se explica a partir de las diferentes esferas de causalidad, accionando en un largo periodo de tiempo. Por otra parte, López Mora, utiliza el territorio del río Tlalnepantla, para comprender procesos más amplios; al hacer confluir en él, multiplicidad de dinámicas económicas, sociales y políticas. En PrazeresVasconcelos y Ferreira-Marta, el río no es accesorio, sino un elemento clave en el proceso de constitución del entramado urbano. Finalmente, en Garnero, las ideas de afirmación de proyectos nacionales y de consolidación territorial, se traducen en enormes iniciativas de transformación material, en las que los espacios fluviales cumplen un rol protagónico.

\section{Consideraciones finales}

En definitiva, todas las concepciones repasadas y la forma en que la mayoría se plasma en los trabajos sobre ríos latinoamericanos, reafirman de forma más taxativa, aspectos tradicionalmente postulados por la historia

\footnotetext{
80. Boelens et al., 2016, 2. Menga y Swyngedouw, 2018, 4.

81. Robbins, Hintz, y Moore, 2014, 131. Fischer-Kowalski y Erb, 2016, 54. Wesselink, Kooy, y Warner, 2017, 2.

82. Bakker y Bridge, 2006, 19. Budds y Hinojosa, 2012, 124. Boelens et al., 2016, 4. Obertreis et al., 2016, 172.
} 
Figura 1: Dinámicas Socio-naturales en Sistemas Fluviales

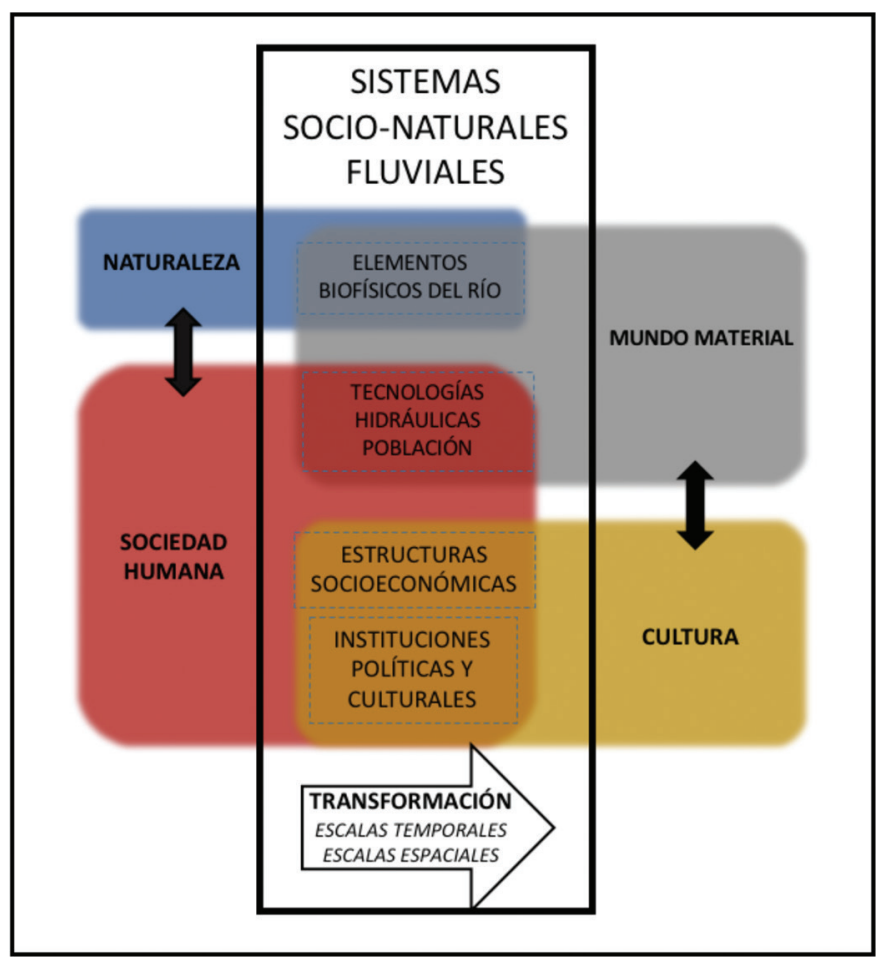

Elaboración propia, basado en: ${ }^{83}$

ambiental. El mundo biofísico, es más que un escenario, trasfondo estático de otros acontecimientos y más que el entorno espacial de la gradual evolución humana ${ }^{84}$. Por el contrario, es coautor de la historia, puesto que esta no puede explicarse sin tener en cuenta este tipo de fenómenos. En consecuencia, tampoco puede entenderse el presente, que siempre es resultado de estructuras y eventos pretéritos ${ }^{85}$.

El presente dossier, representa una maravillosa oportunidad para pensar juntos las similitudes y particularidades de la historia de ríos en América Latina, en dos sentidos. Desde un punto de vista metodológico, ofrece varios tipos de enfoque, diversidad de fuentes y formas particulares de construir nuestras narrativas, sobre estas interacciones recíprocas entre dinámicas sociales e hidrológicas. El repaso de estas conceptualizaciones y el estudio de los casos específicos que aquí presentamos, puede colaborar en proporcionar un lenguaje específico, para analizar fenómenos históricos complejos y para transmitir las interpretaciones que sobre estos hacemos los historiadores.

\footnotetext{
83. Fischer-Kowalski y Haberl, 2007. Menga y Swyngedouw, 2018. Winiwarter y Schmid, 2020.

84. Winiwarter et al., 2016, 151.

85. Winiwarter et al., 2016, 162.
}

Por otro lado, nos permiten analizar cómo la diversidad de espacios ecológicamente diversos y las diferencias en la conformación histórica de aquellos espacios nacionales, dieron como resultado formas de interacción con el agua emparentadas, pero no siempre coincidentes. Además, son un elemento adicional para facilitar la generación de análisis comparativos de mayor profundidad entre ríos en nuestro continente y para explicitar algunos supuestos teóricos en su historiografía, que muchas veces quedan implícitos ${ }^{86}$. Finalmente, esperamos que este esfuerzo colectivo, sirva para resaltar la necesidad de multiplicar y profundizar el estudio del importante y activo rol que han tenido los ríos, en los procesos de construcción de socio-naturalezas en Latinoamérica.

\section{BIBLIOGRAFÍA}

Agnoletti, M. y Neri Sermeri, S. 2014: The basic environmental history. Cham (Switzerland), Springer. https://doi. org/10.1007/978-3-319-09180-8

Armiero, M. 2008: "Seeing like a protester: nature, power, and environmental struggles". Left History 131. https://doi. org/10.25071/1913-9632.24610

Arnold, D. y Roberto, E. 2000: La naturaleza como problema histórico: el medio, la cultura y la expansión de Europa. Ciudad de México (México), Fondo de Cultura Económica.

Arruda, G. 2015: "Bacias hidrográficas, história ambiental e temporalidades". Revista de História Regional 20(2), 209-231.https:// doi.org/10.5212/Rev.Hist.Reg.v.20i2.0001

Bakker, K. y Bridge, G. 2006: "Material worlds? Resource geographies and the matter of nature"'. Progress in human geography 30(1), 5-27. https://doi.org/10.1191/0309132506ph5880a

Bakker, K. 2012: "Water: Political, biopolitical, material". Social Studies of Science 42(4), 616-23. https://doi.org/ 10.1177/0306312712441396

Banister, J. M. 2014: "Are you Wittfogel or against him? Geophilosophy, hydro-sociality, and the state". Geoforum 57, 205-214. https://doi.org/10.1016/j.geoforum.2013.03.004

Blackbourn, D. 2011: "Environmental History and Other Histories". RCC Perspectives, 3,19-21. http://www.environmentandsociety.org/sites/default/files/2011_3.pdf

Boelens, R, Hoogesteger, J. Swyngedouw, E., Vos, J. y P. Wester. 2016: "Hydrosocial territories: a political ecology perspective". Water International, 41 (1), 1-14. https://doi.org/10.10 80/02508060.2016.1134898

\footnotetext{
86. Pritchard, 2013, 7.
} 
Brenner, N. 1998: "Between fixity and motion: accumulation, territorial organization and the historical geography of spatial scales". Environment and planning D: Society and space 16(4), 459-481. https://doi.org/10.1068/d160459

Budds, J., y Hinojosa, L. 2012: "Restructuring and Rescaling Water Governance in Mining Contexts: The Co-Production of Waterscapes in Peru". Water Alternatives 5(1), 119-137. https:// www.water-alternatives.org/index.php/alldoc/articles/vol5/ v5issue1/161-a5-1-8/file

Cabral, D. de C. 2007: "A bacia hidrográfica como unidade de análise em história ambiental". Revista de História Regional 12(1), 133-162. https://revistas2.uepg.br/index.php/rhr/article/ view/2241/1725.

Castro, G. 1995: Los Trabajos de Ajuste y Combate: naturaleza y sociedad en la historia de América Latina. La Habana (Cuba), Casa de las Americas.

Cohen, A., y Davidson, S. 2011: "The watershed approach: Challenges, antecedents, and the transition from technical tool to governance unit". Water alternatives 41(1), 1-14. https://www. water-alternatives.org/index.php/allabs/123-a4-1-1/file

Cronon, W. 1992: "A place for stories: Nature, history, and narrative". Journal of American history 78(4), 1347-1376. https://doi. org/10.2307/2079346

Cronon, W. 1993: "Los Usos de la Historia Ambiental". History Review 17(3), 1-22. https://doi.org/10.2307/3984602

Febvre, L. 2004. El Rin: Historia, mitos y realidades. Ciudad de México (México), Siglo XXI.

Ferreyra, A. I. 2017: "El agua como factor de conflicto y determinante en el precio de la tierra: Córdoba, Argentina, 1800-1855". Agua y Territorio / Water and landscape 10, 30-42. https://doi. org/10.17561/at.10.3607

Fischer-Kowalski, M., y Haberl, H. 2007: "Conceptualizing, observing and comparing socioecological transitions". Socioecological transitions and global change: Trajectories of social metabolism and land use, 1-30. https://doi. org/10.4337/9781847209436

Fischer-Kowalski, M., y Erb, K. 2016: "Core Concepts and Heuristics", in Haberl, H., Fischer-Kowalski, M., Krausmann, F. \& Winiwarter, V. (Eds.), Social Ecology. Society-Nature Relations across Time and Space. Cham (Switzerland), Springer, 29-61. https://doi.org/10.1007/978-3-319-33326-7_2

Fischer-Kowalski, M. y Weisz, H. 2016: "The Archipelago of Social Ecology and the Island of the Vienna School", in Haberl, H., Fischer-Kowalski, M., Krausmann, F. \& Winiwarter, V. (Eds.), Social Ecology. Society-Nature Relations across Time and Space. Cham (Switzerland), Springer, 3-28. https://doi. org/10.1007/978-3-319-33326-7__
Frioux, S. 2014: "Environmental History of Water Resources", in Agnoletti, M. \& Neri Serneri, S. (Eds), The Basic Environmental History. Environmental History. Cham (Switzerland), Springer, 121-41. https://doi.org/10.1007/978-3-319-09180-8_4

Gallini, S. 2004: "Problemas de método en la historia ambiental de América Latina". Anuario IEHS: Instituto de Estudios histórico sociales, 19: 147-72. http://anuarioiehs.unicen.edu.ar/ Files/2004/Problemas\%20de\%20m\%C3\%A9todos\%20en\%20 la\%20historia\%20ambiental\%20de\%20Am\%C3\%A9rica\%20Latina.pdf

Gallini, S. 2012. "La naturaleza cultural de la historia ambiental y su rematerialización"en Hering Torres, M. S. y Pérez Benavides, A. C. (Eds.), Historia Cultural desde Colombia. Categorías y debates, Bogotá (Colombia), Universidad Nacional de Colombia. Universidad Javeriana, Universidad de los Andes, 377-397.

Gierlinger, S., Hauer, F., Pollack, G. y Krausmann, F. 2016: "Metabolismo y paisaje acuático en una ciudad en la industrialización: una evaluación cuantitativa del uso de los recursos y su relación con la transformación del paisaje acuático urbano en Viena del siglo XIX". Agua y Territorio / Water and Landscape, 7, 109-124. https://doi.org/10.17561/at.v0i7.2966

Gómez-Espín, J. M. 2019: "Modernización de regadíos en España: experiencias de control, ahorro y eficacia en el uso del agua para riego". Agua y Territorio / Water and Landscape, 13, 69-76 https://doi.org/10.17561/at.13.3972

González de Molina, M. y Toledo, V. M. 2014. The Social Metabolism. A Socio-Ecological Theory of Historical Change. Cham (Switzerland), Springer. https://doi.org/10.1007/978-3-31906358-4

Hamlin, C. 2000: "Waters' or 'Water'? - master narratives in water history and their implications for contemporary water policy". Water Policy, 2 (4-5), 313-25. https://doi.org/10.1016/S13667017(00)00012-X

Hassan, F. A. 2010: Water history for our times. Paris (France), UNESCO. https://unesdoc.unesco.org/ark:/48223/pf0000210879

Hughes, J. D. 2009: An Environmental History of the World: Humankind's Changing Role in the Community of Life. London (United Kingdom), Routledge. https://doi.org/10.4324/9780203885758

Hunt, R. C. 2009: "Sistemas de riego por canales: tamaño del sistema y estructura de la autoridad", en Palerm, J. y Martínez Saldaña, T. (Eds.), Aventuras con el agua. La Administración del agua de riego: historia y teoría. Texcoco (México), Colegio de Posgraduados, 47-78.

Jakobsson, E. 2002: "Industrialization of rivers: A water system approach to hydropower development". Knowledge, Technology \& Policy 14, 41-56. https://doi.org/10.1007/s12130-002-1014-0 
Kaika, M. 2006: "Dams as symbols of modernization: The urbanization of nature between geographical imagination and materiality". Annals of the Association of American Geographers 96 (2), 276-301. https://doi.org/10.1111/j.1467-8306.2006.00478.x

Krausmann, F., Weisz, H. y Eisenmenger, N. 2016: "Transitions in sociometabolic regimes throughout human history", in $\mathrm{Ha}$ berl, H., Fischer-Kowalski, M., Krausmann, F. \& Winiwarter, V. (Eds.), Social Ecology. Society-Nature Relations across Time and Space. Cham (Switzerland), Springer, 63-92. https://doi. org/10.1007/978-3-319-33326-7_3

Latta, A. y Sasso. J. 2014: "Megaproyectos hídricos y conflicto socio-ambiental en Latinoamérica: perspectivas teóricas desde Gramsci y Foucault". Agua y Territorio / Water and Landscape, 4,70-83. https://doi.org/10.17561/at.v1i4.2166

Le Billon, P. 2015: "Environmental Conflict", in Perreault, T., Bridge, G. \& McCarthy, J. (Eds.), The Routledge Handbook of Political Ecology. London (United Kingdom), Routledge, 598-608.

Leff, E.: 2005: "Vetas y Vertientes de La Historia Ambiental Latinoamericana: una nota metodológica y epistemológica". Varia Historia, 21 (33), 17-31. https://doi.org/10.1590/S010487752005000100002

Linton, J., y Budds, J. 2014: "The hydrosocial cycle: Defining and mobilizing a relational-dialectical approach to water". Geoforum, 57, 170-80. https://doi.org/10.1016/j.geoforum.2013.10.008

Lopez Mora, R. 2019: "El Abastecimiento de agua y saneamiento urbano". Agua y Territorio / Water and Landscape, 14, 133-134. https://doi.org/10.17561/at.14.4978

Loreto López, R. 2016: "Escasez, guerras y desigualdad social. El proyecto modernizador del sistema de abasto hídrico de una ciudad mexicana: Puebla, siglos XVII-XX". Agua y Territorio / Water and Landscape, 7, 75-91. https://doi.org/10.17561/at.v0i7.2964

Martos-Núñez, E., y Martos-García, A. 2015: "Memorias e imaginarios del agua: nuevas corrientes y perspectivas". Agua y Territorio / Water and Landscape, 5, 121-131. https://doi. org/10.17561/at.v0i5.2539

Matés Barco, J. M. 2013: "La conquista del agua en Europa: los modelos de gestión (siglos XIX y XX)". Agua y Territorio / Water and Landscape, 1, 21-29. https://doi.org/10.17561/at.v1i1.1030

Mauch, C. y Zeller, T. 2008: "Rivers in history and historiography: an introduction", in Mauch, C. \& Zeller, T. (Eds.), Rivers in history: perspectives on waterways in Europe and North America. Pittsburgh (United States), University of Pittsburgh Press, 1-10.

McCully, P. 2004: Ríos silenciados: ecología y política de las grandes represas. Buenos Aires (Argentina), Proteger.

McNeill, J. R. 2000: Something new under the sun: an environmental history of the twentieth-century world. New York (United States), Norton.
McNeill, J. R. 2005: "Naturaleza y cultura de la historia ambiental". Nómadas, 22, 12-22. http://nomadas.ucentral.edu.co/ nomadas/pdf/nomadas_22/22_1M_Naturalezayculturadelahistoria.pdf

Menga, F., y Swyngedouw, E. 2018: "States of Water", in Menga, F. \& Swyngedouw, E. (Eds.). Water, Technology and the NationState. London (United Kingdom), Routledge, 1-18. https://doi. org/10.4324/9781315192321-1

Molle, F. 2009: "River-basin planning and management: The social life of a concept". Geoforum, 40 (3), 484-94. https://doi. org/10.1016/j.geoforum.2009.03.004

Molle, F., Mollinga, P. P. y Wester, P. 2009: "Hydraulic Bureaucracies: Flows of Water, Flows of Power". Water Alternatives 2 (3), 328-475. https://www.water-alternatives.org/index.php/alldoc/ articles/vol2/v2issue3/65-a2-3-3/file

Molle, F. y Wester P. 2009: "River basin trajectories: An inquiry into changing waterscapes", in Molle, F. \& Wester, P. (Eds.) River basin trajectories: societies, environment and development. Wallingford (United Kingdom), CAB Internacional, 1-19. https:// doi.org/10.1079/9781845935382.0001

Mosley, S. 2010: The environment in world history. London (United Kingdom), Routledge. https://doi.org/10.4324/9780203859537

Norman, E. S., Bakker, K. y Cook, C. 2012: "Introduction to the Themed Section Water Governance and the Politics of Scale". Water Alternatives, 5 (1), 52-61. https://www. water-alternatives. org/index.php/alldoc/articles/vol5/v5issue1/157-a5-1-4/file

Obertreis, J., Moss, T. Mollinga, P. y Bichsel, C. 2016: "Water, infrastructure and political rule: Introduction to the special issue". Water Alternatives 9 (2), 168-181. https://www.wateralternatives.org/index.php/alldoc/articles/vol9/v9issue2/311a9-2-1/file

Palerm, J. y Martínez Saldaña, T. (Eds.) 2012: Aventuras con el agua. La Administración del agua de riego: historia y teoría. Texcoco (México), Colegio de Posgraduados

Pritchard, S. B. 2013: "Joining environmental history with science and technology studies: promises, challenges, and contributions", in Jorgensen, D., Jorgensen, F. A. \& Pritchard, S. B. (Eds.), New Natures: Joining Environmental History with Science and Technology Studies. Pittsburgh (United States), University of Pittsburgh Press, 1-18. https://doi.org/10.2307/j.ctt5vkgkn.4

Radkau, J. 2008: Nature and Power: A Global History of the Environment, Cambridge (United Kingdom), Cambridge University Press.

Rattu, P. y Véron, R. 2016: "Towards a Foucauldian Urban Political Ecology of water: Rethinking the hydro-social cycle and scholars' critical engagement". Foucault Studies, 21, 138-158 https://doi.org/10.22439/fs.v0i0.5021 
Rautenen, S., Luosi, A. Nygard, H. Vuorinen, H. S. y Rajala, R. P. 2010: "Sanitation, water and health". Environment and History 16 (2), 173-194. https://doi.org/10.3197/09673401 OX12699419057250

Robbins, P. 2011: Political Ecology: A Critical Introduction. Hoboken (United States), John Wiley \& Sons.

Robbins, P., Hintz, J. y Moore, S. A. 2014: Environment and Society: A Critical Introduction. Hoboken (United States) John Wiley \& Sons.

Rückert, F. Q. 2017: "O abastecimento de água na perspectiva da historiografia europeia e hispano-americana". Revista História: Debates e Tendências 17 (1), 157-179. https://doi.org/10.5335/ hdtv. 17n. 1.7241

Scarpino, P. V. 2014: "A historian's perspective on rivers of the Anthropocene", in Bhaduri, A., Bogardi, J., Leentvaar, J. \& Marx, S. (Eds.). The Global Water System in the Anthropocene. Cham (Switzerland), Springer, 161-171. https://doi.org/10.1007/978-3319-07548-8_11

Schmidt, J. J. 2014: "Historicising the hydrosocial cycle". Water Alternatives, 7 (1), 220-234. https://www. water-alternatives.org/ index.php/alldoc/articles/vol7/v7issue1/242-a7-1-13/file

Schönach, P. 2017: "River histories: a thematic review". Water History, 9, 233-257. https://doi.org/10.1007/s12685-016-0188-4

Scott, J. C. 1998: Seeing like a state: How certain schemes to improve the human condition have failed. New Haven (United States), Yale University Press.

Simmons, I. G. 2008: Global Environmental History: 10,000 BC to $A D$ 2000. Edinburgh (United Kingdom), Edinburgh University Press. https://doi.org/10.3366/edinburgh/9780748621583. 001.0001

Smith, N. 1994: Uneven Development: Nature, Capital, and the Production of Space. Athens (United States), University of Georgia Press.

Solomon, S. 2010: Water: The Epic Struggle for Wealth, Power, and Civilization. New York (United States), Harper Collins.

Soluri, J., Leal, C. \& Pádua, J. A. 2018: A Living Past: Environmental Histories of Modern Latin America. New York (United States), Berghahn Books. https://doi.org/10.2307/j.ctvw04gzn

Sörlin, S. y Warde, P. 2005: The Problem of the Problem of Environmental History- a Re-reading of the Field and Its Purpose (Conference). Cambridge (United Kingdom), Centre for History and Economics, University of Cambridge. https://www.histecon. magd.cam.ac.uk/envdoc/uses_env/documents.htm

Stewart, M. A. 1998: "Environmental History: Profile of a Developing Field". The History Teacher, 31 (3), 351-368. https://doi. org/10.2307/494883

Swyngedouw, E. 1999: "Modernity and Hybridity: Nature, Regeneracionismo, and the Production of the Spanish Waterscape, 18901930". Annals of the Association of American Geographers, 89 (3), 443-465. https://doi.org/10.1111/0004-5608.00157
Swyngedouw, E. 2009: "The Political Economy and Political Ecology of the Hydro-Social Cycle". Journal of Contemporary Water Research \& Education, 142 (81), 56-60. https://doi.org/10.1111/ j.1936-704X.2009.00054.X

Swyngedouw, E. 2010: Place, nature and the question of scale: Interrogating the production of nature. Berlin (Germnay), BerlinBrandenburgische Akademie der Wissenschaften.

Tvedt, T. 2010: ' Water Systems', Environmental History and the Deconstruction of Nature". Environment and history, 16 (2), 143166. https://doi.org/10.3197/096734010X12699419057179

Urquijo, P. S., Vieyra, A. y Bocco, G. (Coords.) 2017: Geografía e Historia Ambiental. Ciudad de México (México), Universidad Autónoma de México, Centro de Investigaciones en Geografía Ambiental (CIGA-UNAM). https://doi.org/10.22201/ ciga.9786070295669p.2017

Walker, R. 2011: «On the Edge of Environmental History». RCC Perspectives, 3: 48-52. http://www.environmentandsociety.org/ sites/default/files/2011_3.pdf

Wesselink, A., Kooy, M. y Warner, J. 2017: "Socio-Hydrology and Hydrosocial Analysis: Toward Dialogues across Disciplines". WIREs Water, 42, e1196. https://doi.org/10.1002/wat2.1196

Wester, P. 2009: "Capturing the waters: the hydraulic mission in the Lerma-Chapala Basin, Mexico (1876-1976)". Water History, 11, 9-29. https://doi.org/10.1007/s12685-009-0002-7

Winiwarter, V. y Schmid, M. 2020: "Socio-Natural Sites", in Haumann, S., Knoll, M. \& Mares, D. (Eds.), Concepts of Urban-Environmental History, Bielefeld (Germnay), transcript Verlag, 33-50. https://doi.org/10.14361/9783839443750-003

Winiwarter, V., Schmid, M. y Dressel, G. 2013: "Looking at half a millennium of co-existence: the Danube in Vienna as a socionatural site". Water History, 5, 101-119. https://doi.org/10.1007/ s12685-013-0079-X

Winiwarter, V., Schmid, M., Haberl, H. y Singh, S. J. 2016: "Why legacies matter: Merits of a long-term perspective", in Haberl, H., Fischer-Kowalski, M., Krausmann, F. \& Winiwarter, V. (Eds.), Social Ecology. Society-Nature Relations across Time and Space. Cham (Switzerland), Springer, 149-168. https://doi. org/10.1007/978-3-319-33326-7_6

Wittfogel, K. 1957: Oriental despotism: A study of total power. New Haven (United States), Yale University Press.

Worster, D. 1992: Rivers of Empire: Water, Aridity, and the Growth of the American West. Oxford (United Kingdom), Oxford University Press. 